\title{
ANÁLISIS DE LA EXPANSIÓN DE REDES DE ACCESO PASIVAS DE FIBRA ÓPTICA GPON Y BPON EN LA CIUDAD DE MEDELLÍN
}

\author{
Alejandro Correa-Muñoz ${ }^{1}$ \\ Claudia M. Serpa-ImbetT ${ }^{2}$
}

\section{Resumen}

En este trabajo se reporta un análisis de la expansión de Redes de Acceso Pasivas de Fibra Óptica a partir de mediciones de potencia óptica y de reflectometría en el dominio del tiempo en una red instalada en la ciudad de Medellín. Se hizo el análisis entre uno de los usuarios de la red y la Oficina Central a una distancia de $1,2 \mathrm{~km}$, y se encontró una potencia en la terminal de red óptica de $-18,8 \mathrm{dBm}$ que es adecuada para los servicios demandados por el usuario. Para la migración a nuevas tecnologías de transmisión en Redes de Acceso Pasivas se recomienda el análisis de la atenuación espectral en la fibra instalada, el cambio en la razón de división de potencia (split ratio) de los divisores ópticos y el ajuste de niveles de potencia en las terminales ópticas de red, con el fin de permitir la expansión gradual para nuevas instalaciones de usuarios desde la Oficina Central. Se propone el análisis de implementación de otras topologías con el fin de potencializar el crecimiento y la adopción gradual de nuevas tecnologías como las Redes Pasivas Multiplexadas por longitud de Onda.

1 Departamento de Mantenimiento, Planta Externa Línea Comunicaciones S.A., alejandrocm25@hotmail.com.

2 Centro de Investigación, Instituto Tecnológico Metropolitano, claudiaserpa@itm. edu.co 
[60] Análisis de la expansión de redes de acceso pasivas de fibra óptica gpon y bpon...

\section{Palabras clave}

Red de Acceso Pasiva, GPON, BPON, multiplexor, divisor óptico.

\section{Abstract}

In this work, we present an analysis of the Optical Passive Fiber Network by measurement of optical power and Optical Reflectometry in the time domine on a network installed in Medellín city. We made the analysis between one network's user and Central Office at $1,2 \mathrm{~km}$ of distance and $-18,8 \mathrm{dBm}$ of optical power was measured. This value is adequate for user service demanded. For new technology migration in Passive Optical Network the analysis of spectral attenuation over installed fiber, the change in splitter ratio and adjust of optical power are recommended. This analysis will allow the gradual expansion for news installation of users from the Central Office. This analysis is a proposal for topology implementation caused by the growth and new technology adoption such as passive wavelength multiplexing.optical network.

\section{Keywords}

Optical access network, GPON, BON, Multiplexor, splitter. 


\section{INTRODUCCIÓN}

Las Redes Óptica Pasivas (PON, Passive Optical Network) son redes punto-multipunto que remplazan los componentes activos entre la Unidad Óptica de Red (ONU, Optical Network Unit) y la Terminal de Línea Óptica (OLT, Optical Line Terminal) por componentes ópticos pasivos como el Divisor Óptico y el Multiplexor Óptico para guiar el tráfico (Elbers, 2010). La utilización de estos sistemas pasivos reduce los costos y mantenimiento de la red, además ayudan a resolver el problema de los cuellos de botella producidos por las redes de acceso que soportan el ancho de banda requerido para la transmisión de triple-play: datos, voz y video, demandados por los usuarios finales. (Chanclou et al., 2008).

Las PON son la clave para dar respuesta a la creciente demanda de los servicios de banda ancha. Este crecimiento requiere de la migración de una tecnología de transmisión hacia otra (por ejemplo de BPON hacia GPON) para responder con la velocidad adecuada a los usuario finales. En general, en la migración de tecnologías de transmisión basadas en PON se deben adecuar las condiciones físicas de la red instalada, ya que el cambio de longitud de onda de las fuentes de luz de los MUX produce valores diferentes de la atenuación espectral en la fibra instalada y niveles de potencia diferente en las ONT, así como cambios en la razón de división de potencia (split ratio) de los divisores ópticos (Agrawal, 2007). El análisis de estas condiciones permite una optimización adecuadamente los recursos usados la red para posibilitar su crecimiento gradual, la flexibilidad al cambio de arquitecturas y la expansión a gran escala de la infraestructura instalada que permita avanzar en las próximas demandas de requerimiento de ancho de banda (Kim, 2003; Chang et al., 2009).

El uso de las PON está direccionado a crear troncales de velocidad de acceso menor en donde converge el tráfico de redes locales. Las topologías tradicionales para las redes ópticas son el anillo y la estrella, sin embargo últimamente existe una controversia acerca de las posibilidades de tener una red con una topología 
que permita elevar el nivel de confiabilidad y recuperación ante los errores (mesh networks) o redes de supervivencia (survability networks). Independientemente de la topología y los mecanismos de supervivencia de la red, las arquitecturas propuestas deben enfrentar dos de los problemas en el desarrollo de nuevas tecnologías, la compatibilidad hacia atrás y la posibilidad de un crecimiento y adopción gradual (Sivalingam, 2005).

En este trabajo se presenta un análisis del crecimiento y migración de una red de acceso pasiva BPON hacia una GPON. Para este propósito, se analizan los registros de potencia óptica y OTDR en un usuario en la red cuyas características muy son similares a los de otros usuarios. Finalmente, se encuentra que la potencia óptica que llega a la Terminal de Red Óptica (ONT, Optical Network Terminal) es de $\sim 18 \mathrm{dBm}$, y de acuerdo con la sensitividad de los detectores y los estándares para redes BPON y GPON es posible bajar hasta $\sim 10 \mathrm{~dB}$ más para posibilitar mayores alcances en la distribución o la adición de otros usuarios. El objetivo de ésta CO (CO, Central Office) es cubrir la ciudad de Medellín con una red GPON, para cumplir con la recomendación de usar el menor número de $\mathrm{CO}$ en las redes de acceso pasivas de las áreas Metropolitana (Chanclou et al., 2008), con este propósito, este análisis propone recomendaciones de los cambios graduales que se deben hacer la arquitectura del tendido para potencializar el uso de los recursos de la red existente.

\section{Redes ópticas pasivas (PON)}

La Fig. 1, muestra una arquitectura básica de una red óptica pasiva que está formada básicamente por una OLT que se encuentra en CO, un Divisor Óptico (Spliter), y varias Unidades de Red Óptica (ONU, Optical Network Unit) formadas por ONT ubicadas muy cerca a los usuarios finales y la transmisión se realiza entre la OLT y la ONU a través del Divisor Óptico, que direcciona el tráfico bidireccionalmente dependiendo si el canal es ascendente o descendente (Kazovski, 2007). 


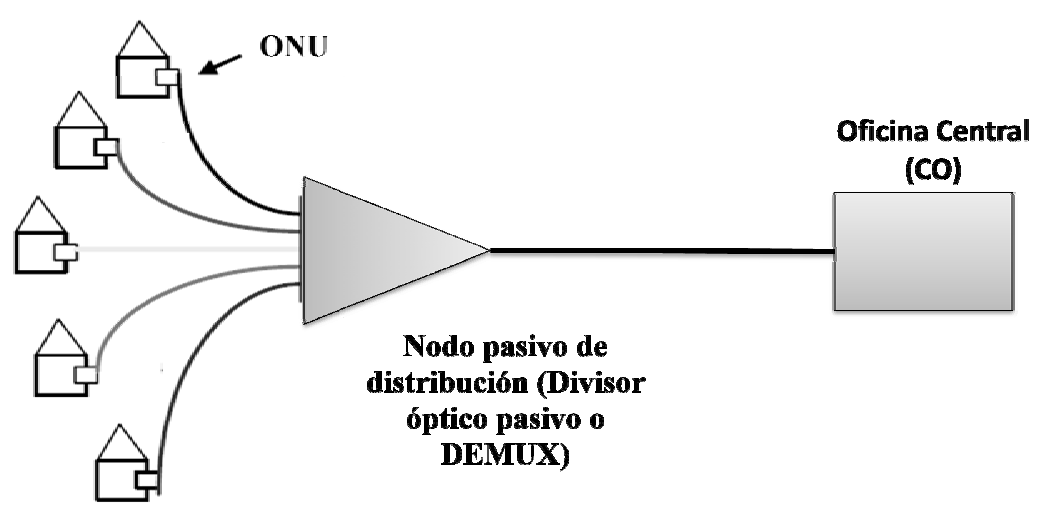

Fig. 1. Arquitectura básica de una PON (Mastrodonato, 2005)

La ITU (International Telecommunication Unión) generó el estándar G.983 para APON y BPON que permite una velocidad a 622 Mbps downstream y 155 Mbps upstream y G.984 para GPON's. La IEEE (The Institute of Electrical and Electronics Engineers) dentro de los desarrollos de EFM (Ethernet in the First Mile) generó los estándares para EPON's: IEEE 802.3ah para que sea compatible con cualquier red del estándar IEEE 802.3. La norma G.984 posibilita la explotación de las redes GPON hasta regímenes de $2488 \mathrm{Mbps}$, soportando los protocolos Ethernet, ATM (Asynchronous Transport Mode) y TDM. Este ultimo soporta, de forma directa, tanto servicios síncronos (voz y vídeo, por ejemplo, mediante TDM) como asíncronos (datos, por ejemplo, vía ATM), al contrario, una red EPON únicamente admite el modo de transporte Ethernet, y, consecuentemente, cualquier servicio soportado por dicho protocolo (ITU-T, 2010).

En el canal ascendente las ONU transmiten contenidos a la OLT por lo que es necesario el uso de Acceso por División de Tiempo (TDMA, Time Division Multiplexing Access) para las GPON y Protocolo de Control de Múltiples puntos (MPCP, MultiPoint Control Protocol) para EPON. Para evitar interferencias entre el canal descendente y ascendente se utilizan dos longitudes de onda 
insertadas en el mismo hilo de fibra usando un multiplexor óptico (MUX) y filtros ópticos para separarlas después. Para el diseño y distribución de las PON es muy importante considerar la distancia entre usuario y la CO. Un usuario cercano a la CO necesitará una potencia menor, mientras que un usuario lejano necesitará una potencia mayor. La potencia óptica es un parámetro importante en la minimización del despliegue de fibra óptica y la maximización la distancia entre el usuario final y la CO que es de hasta $20 \mathrm{~km}$ para GPON y $10 \mathrm{~km}$ para BPON (Sivalingam, 2005).

\section{MontAJE EXPERIMENTAL}

La Fig. 2 muestra la arquitectura de la red óptica pasiva (PON, Passive Optical Network) de acceso el cual consiste de un Multiplexor Óptico (MUX-OLT) de referencia F152 Hi-FOCuS ${ }^{\mathrm{TM}} 5$ para funcionamiento bajo el estándar GPON, este MUX-OLT permite un alcance hasta el usuario final de hasta $40 \mathrm{~km}$ usando fibra monomodo 1000BASE-LX-E operando a $1310 \mathrm{~nm}$ con receptores ópticos al final del enlace de sensitividad de $-22 \mathrm{dBm}$ ) (Al-Azzawi, 2007). El MUX-OLT opera a $1490 \mathrm{~nm}$ para enviar información hacia el cliente (downstream) y a $1310 \mathrm{~nm}$ para recibir información desde los clientes (upstream). Este es conectado a un distribuidor de fibra óptica (ODF, Optical Distributor Fiber) a través de un patchcord FC/APC. El MUX-OLT y el ODF son la Oficina Central (CO, Central Office) de la PON. La CO está conectada a un hilo de fibra óptica monomodo (SMF, singlemode fiber) de $0,4 \mathrm{~km}$ que es parte de un cable de fibra óptica de 72 hilos que constituye el backbone para futuras distribuciones de acceso desde la CO.

El final de este hilo está conectado a un divisor de potencia de 1:32 referencia FSASA2-132 Tyco Electronic con una pérdida por inserción en cada terminal de $\sim 15+/$ - $1 \mathrm{~dB}$ a $1310 \mathrm{~nm} \mathrm{y} \sim 15+/-0,5$ $\mathrm{dB}$ a $1550 \mathrm{~nm}$. Este divisor de potencia está conectado a un hilo de 0,28 km parte de un cable de fibra óptica de 72 hilos que se conecta a una caja de distribución de 4 accesos (B4). Uno de los accesos se conecta a un punto de acceso de red al usuario (NOP, Network Access Point) a través de un hilo de fibra de $0,14 \mathrm{~km}$ parte de un 
cable de 36 hilos. El NOP es una caja de distribución de 8 accesos (A8) que distribuyen las señales finalmente a las Terminales de Red Óptica (ONT, Optical Network Terminal) de los usuarios finales a través de un hilo de fibra óptica de $\sim 0,44 \mathrm{~km}$ que es parte de un cable de 12 hilos.

Las ONT que operan bajo el estándar BPON (ONT-BPON) son de referencia Tellabs 1600-612 y reciben información a velocidades $622 \mathrm{Mbps}$ para downstream con niveles de potencia óptica -8 hasta -28 dBm y envían información a $155 \mathrm{Mbps}$ para tráfico upstream a través de la modulación de un laser que emite potencia óptica a $1490 \mathrm{~nm}$ con niveles de +0 hasta $+4 \mathrm{dBm}$ Las ONT que operan bajo el estándar GPON (ONT-GPON) son de referencia Tellabs 1600-709 y reciben información a velocidades 2.488 Gbps para downstream con niveles de potencia óptica -8 hasta $-27 \mathrm{dBm}$ y envían información a 1.244 Gbps para tráfico upstream a través de la modulación de un laser que emite potencia óptica a $1490 \mathrm{~nm}$ con niveles de $+0,5$ hasta $+5 \mathrm{dBm}$. Esta arquitectura tipo bus es comúnmente usada en redes de acceso pasivas por la facilidad de implementación, acceso y mantenimiento. Esta construcción hace parte de la red de acceso operada por una empresa operadora de servicios de la ciudad de Medellín.

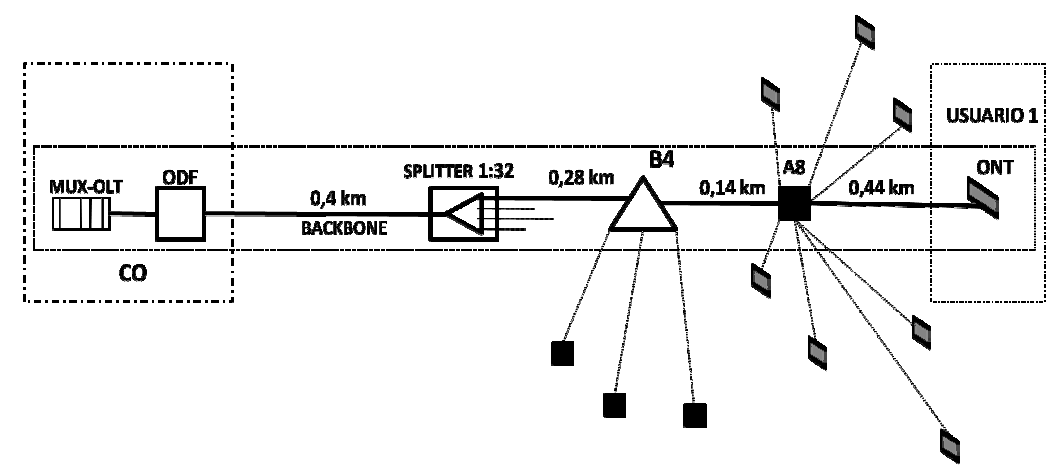

Fig. 2. Montaje experimental de la Red de Acceso GPON y EPON 


\section{RESULtADOS EXPERIMENTALES}

Se usó el OTDR EXFO FTB-300 programado en modo automático a una longitud de onda $1310 \mathrm{~nm}$, para registrar el estado de un acceso desde la terminal del USUARIO 1 hasta A8 como se muestra en la Fig. 3. La distancia registrada fue de $\sim 0,44 \mathrm{~km}$ m y la pérdida aproximada en el tramo de $0,358 \mathrm{~dB}$ debido a la atenuación de la fibra que es de $0,2 \mathrm{~dB} / \mathrm{km}$ y las pérdida por inserción de conectorizaciones intermedias.

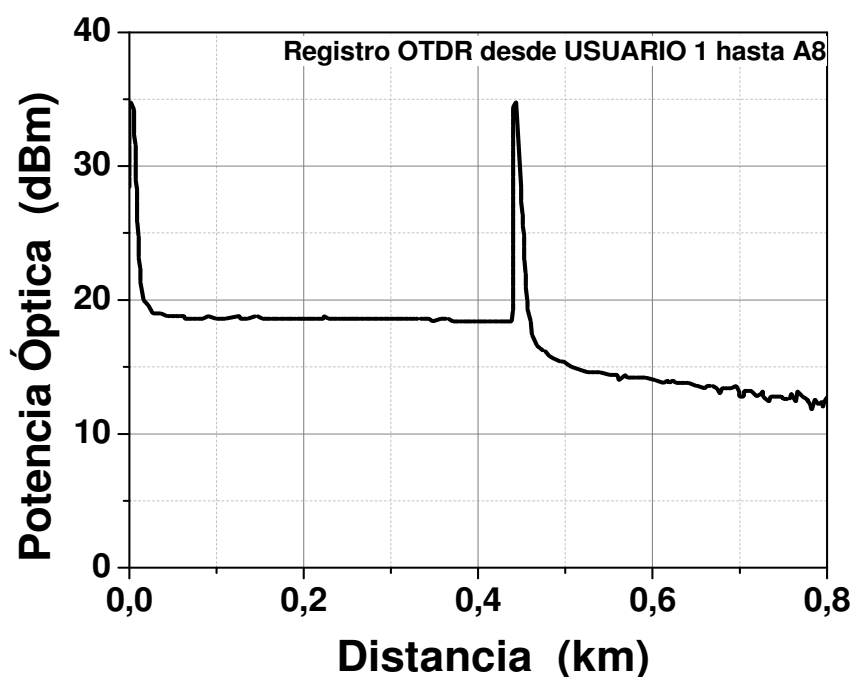

FIG. 3. REgISTRO DESDE USUARIO 1 HASTA EL A8 USANDO EL OTDR

Se usó un detector FPM300 para medir el nivel de potencia en cada uno de los tramos de la red a $1310 \mathrm{~nm}$. La potencia registrada entre el MUX-OLT y el divisor de potencia 1:32 fue de -16,6 dBm, entre el MUX-OLT y el B4 fue de -17,3 dBm, entre el MUX-OLT y A8 fue de -18,4 dBm y entre el MUX-OLT y la ONT que llega al Usuario A (Ver Fig. 1 y 3) fue de -18,8 dBm. Los valores de pérdida por tramo oscilan entre 0,4 y $1,1 \mathrm{~dB}$, y son aceptables teniendo en cuenta la distancia entre los equipos intermedios como se muestra en la Fig. 4. 
Adicionalmente el nivel de potencia que llega a la OLT del USUARIO $1 \mathrm{de}-18,8 \mathrm{dBm}$ es adecuado para la sensitividad del fotodetector de la ONT. El uso de las terminales receptoras ONTBPON o ONT-GPON está determinado por el tipo el protocolo de transmisión usado en la red de acceso pasiva: BPON o GPON.

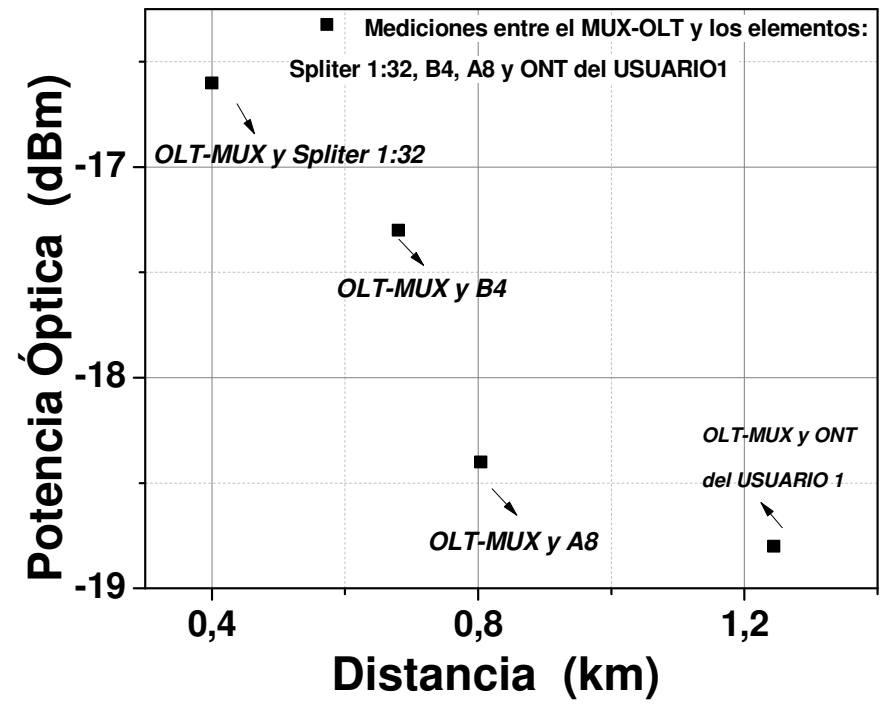

Fig. 4. Potencia Óptica entre el muX-Olt y CAdA uno de los elementos de LA Red

\section{Discusión}

Se verificó el estado del acceso del USUARIO 1 desde la terminación de fibra hasta la A8 a través del registro del OTDR que mostró un enlace continuo sin roturas o conectorizaciones de baja calidad y con un valor de pérdida dentro de lo aceptable para una distancia de $0,4 \mathrm{~m}$. La potencia óptica que llega al USUARIO 1 es de 18,8 dBm. Dada la la sensitividad de los fotodetectores de las ONT es posible bajar la potencia $\sim 10 \mathrm{dBm}$ adicionales, para expandir y distribuir los accesos de la red hasta el límite que recomiendan los estándares que es de $10 \mathrm{~km}$ para una red BPON y 20 a $60 \mathrm{~km}$ para una red GPON respectivamente (ITU-T, 2010). 
El MUX-OLT de esta red de acceso tiene posibilidades de expansión a partir del uso de los 71 hilos restantes del cable backbone o del rediseño de la arquitectura de algunos accesos que permitan tener tramos más largos para cubrir lugares más lejanos que los reportados en la Fig. 2. Se propone diseñar la arquitectura de cubrimiento de las áreas analizando mediciones provenientes de detectores de potencia y OTDR y limitando la distancia de la usuario final cuando el nivel de potencia óptica en las terminales de las ONT sea muy cercanas a la sensitividad de su fotodetector, además de implementar en el crecimiento de la red otro tipo de topologías que permita redireccionar el y proteger el tráfico en caso de fallas.

\section{Conclusiones}

Las red de acceso pasiva analizada es una red de servicios de banda ancha que usa la arquitectura tipo de una red de fibra ya instalada, y migra de estándares BPON hacia GPON mediante el cambio de los equipos terminales MUX-OLT de la OC y ONT de los usuarios finales. El cubrimiento del MUX-OLT analizado en éste trabajo que trabaja bajo el estándar GPON es $20 \mathrm{~km}$, por hilo suficiente cubrir el área de Medellín. En los resultados se presentan las condiciones para la extensión de cada tramo que depende de la cercanía de los usuarios a la OC y la sensitividad de los receptores de las ONT. El análisis muestra las condiciones para adecuar gradualmente la infraestructura de la red de acuerdo al crecimiento que demande la migración hacia nuevas tecnologías de transmisión sobre redes PON de mayor capacidad tales como las multiplexadas por longitud de onda WDM-PON y las redes Ethernet de alta capacidad de transmisión (XG-PON) (Dutta, 2004; Elbers, 2010) que ofrecen mayores prestaciones en enrutamiento $\mathrm{y}$ asignacion de ancho de banda a los usuarios finales. 


\section{Agradecimientos}

Este trabajo se realizó con información suministrada por una Empresa de Proveedora de Servicios basada en redes de acceso PON de la ciudad de Medellín. El nombre de la empresa hace parte de la reserva de información de la cooperación investigativa. Este reporte hace parte de los resultados del proyecto Código: P07203 financiado por el Instituto Tecnologico Metropolitano Institución Universitaria de Medellín-Colombia. Los autores agradecen al Ingeniero José Augusto Córdoba por sus comentarios.

\section{ReFERENCIAS}

Agrawal, G., (2007); Fiber-optic communication systems, Wiley-Interscience, Michigan, USA.

Al-Azzawi, A., (2007); Fiber optics: principles and practices, Optical Science And Engineering Series, Boca Raton Florida, USA.

Allan, W., Snyder, J.D., (2000); Optical Waveguide Theory, Kluwer Academic Publisher. Norwell, USA.

Dutta, N.K., (2004); WDM technologies: Optical Network, Academic Press, New York, USA.

Elbers, J.P., (2010); Optical-Access Solutions Beyond 10G-EPON/XG-PON. In Proceeding Optical Fiber Conference, paper OTUO1, San Diego California, USA.

Chanclou, P., y otros 16 autores (2008); Access network evolution: optical fibre to the subscribers and impact on the metropolitan and home network, C. R. Physique, 9, 935-946.

Chang, G.K., Chowdhury, A., Jia, Z., Chien, H.C., Huang, M.F., Yu, J., Ellinas, G., (2009); Key Technologies of WDM-PON for Future Converged Optical Broadband Access Networks [Invited], Journal Optics Communications Network, 1(4), 35-50.

ITU-T, (2010); www.itu.int, (Consultado 10, 01, 2010), International Union Telecommunication.

Kim, K.S., (2003); On the evolution of PON-based FTTH solutions. Informaton Sciences, 149, 21-30. 
[70] Análisis de la expansión de redes de acceso pasivas de fibra óptica gpon y bpon...

Leonid, G., Kazovski, W.T., Gutierrez, D., Cheng, N., Wong, S.W., (2007); Next-Generation on Optical Access Network (Invited paper. Journal of Lightwave Technology, 25(11), 3428-3442.

Mastrodonato, R., Paltenghi, G., (2005); Analysis of a Bandwidth Allocation Protocol for Ethernet Passive Optical (EPON), Proceeding of 7th International Conference Optical Transparent Network, 241-244, Munich, Germany.

Sivalingam, K., (2005); Emerging optical network technologies: architectures, protocols, and performance, Springer, Washington, USA. 